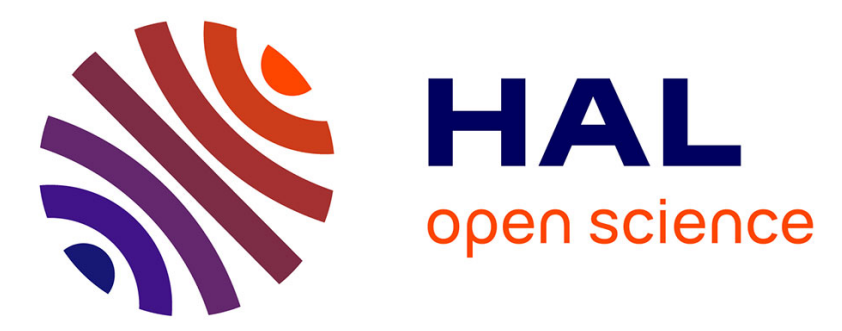

\title{
Comportement du sous-sol soumis à l'influence d'un puits où règne une température imposée. Méthodes de calcul, approche expérimentale
}

A. Baudoin, Michel Abgrall, J. Padet

\section{- To cite this version:}

A. Baudoin, Michel Abgrall, J. Padet. Comportement du sous-sol soumis à l'influence d'un puits où règne une température imposée. Méthodes de calcul, approche expérimentale. Revue de Physique Appliquée, 1988, 23 (3), pp.301-313. 10.1051/rphysap:01988002303030100 . jpa-00245774

HAL Id: jpa-00245774

https://hal.science/jpa-00245774

Submitted on 1 Jan 1988

HAL is a multi-disciplinary open access archive for the deposit and dissemination of scientific research documents, whether they are published or not. The documents may come from teaching and research institutions in France or abroad, or from public or private research centers.
L'archive ouverte pluridisciplinaire HAL, est destinée au dépôt et à la diffusion de documents scientifiques de niveau recherche, publiés ou non, émanant des établissements d'enseignement et de recherche français ou étrangers, des laboratoires publics ou privés. 


\title{
Comportement du sous-sol soumis à l'influence d'un puits où règne une température imposée. Méthodes de calcul, approche expérimentale (*)
}

\author{
A. Baudoin, M. Abgrall et J. Padet \\ Groupe de Thermomécanique, Faculté des Sciences de Reims, Moulin de la Housse, BP 347, 51062 Reims \\ Cedex
}

(Reçu le 16 janvier 1987, révisé le 6 novembre 1987, accepté le 16 décembre 1987)

\begin{abstract}
Résumé. - Dans le cadre d'une étude sur le stockage intersaisonnier de la chaleur en sous-sol par échangeur vertical, nous nous proposons d'établir l'expression du champ de température dans un milieu infini limité intérieurement par un cylindre de longueur également infinie. Nous développons pour cela trois méthodes dites : asymptotique, intégrale et de Gaver. Nous comparons d'abord ces méthodes entre elles pour vérifier leur équivalence, puis nous calons la méthode de Gaver sur une expérimentation grandeur nature. Cette étude nous permet, d'une part, d'obtenir une modélisation bidimensionnelle simple du phénomène dans un cas assez général, et d'autre part, de justifier expérimentalement cette approche bidimensionnelle du problème.
\end{abstract}

\begin{abstract}
In the setting of seasonnal heat storage in ground by vertical heat exchangers, we propose an expression of the temperature field for an infinite surrounding, internally limited by an infinite length cylinder. We develop three methods : asymptotic, integral and Gaver. First we compare each of them with the two others to prove their equivalence, then we carry out an adjustement with scale one experimentation. That study permits, first, to obtain a simple bidimensionnal modelisation of the phenomenon, and then to prove experimentally the bidimensionnal approach of the problem.
\end{abstract}

\section{NOTATIONS UTILISÉES}

\section{$a$}

$k_{\mathrm{p}}$

$k_{\mathrm{p}}^{\prime}$

$p$

$r$

$r^{\prime}$

$R_{\mathrm{p}}$

$t$

$t^{\prime}$

$T$

$T_{\mathrm{a}}$

$T_{\mathrm{p}}$

$\lambda$

$\omega=\sqrt{p / a}$

$\sigma=1,781$ diffusivité du sol

coefficient global d'échange puits-sol

coefficient global d'échange

fonctions de Bessel modifiées de $1^{\text {re }}$ et $2^{\mathrm{e}}$ espèce

variable de Laplace

variable rayon

rayon adimensionnel

rayon de puits

variable temps

temps adimensionnel

température du sol

température dans le puits

température du sol à la paroi

conductivité du sol

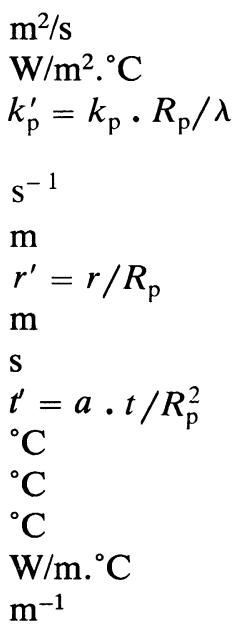

exponentielle de la constante d'Euler $\Gamma=0,577215$

(*) Etude financée par l'Agence Française pour la Maîtrise de l'Energie. Région Champagne-Ardenne. District de Reims.

Réalisée en collaboration avec le BRGM/SGR de Reims. 


\section{Introduction.}

L'intérêt porté actuellement aux systèmes de stockage longue durée de chaleur par batterie de puits verticaux a conduit plusieurs équipes de recherche à se confronter au problème. Différents travaux ont débouché sur des solutions numériques, ou analytiques dans le cas du régime établi [1]. Il apparaît cependant essentiel de connaître la phase transitoire qui va de la mise en fonctionnement du dispositif à son régime établi, si ce dernier est atteint un jour. Ceci afin, entre autres, de déterminer la durée de cette phase transitoire en nombre de cycles. La résolution analytique du problème devrait permettre d'apprécier à sa juste valeur l'intérêt d'une installation de ce type sans recourir à de gros moyens informatiques.

Le premier temps de la recherche de cette solution est l'établissement du champ de température dans le sol environnant un puits unique au cours de la première phase de chauffage. En complément d'autres travaux [2], nous nous plaçons dans le cas le plus général, c'est-à-dire avec un coefficient d'échange à la paroi entre le forage et le sous-sol. Nous imposons alors un échelon de température au fluide dans l'échangeur à l'instant 0 , sachant que la solution pour une température de fluide variable s'obtient par un produit de convolution de la solution trouvée. La résolution se fera d'abord dans l'espace de Laplace, puis, nous revenons à l'espace réel au moyen des trois méthodes : asymptotique, intégrale et de Gaver.

Pour les ordres de grandeur des différents termes, nous nous référons aux valeurs données par une expérimentation en cours.

Nous adoptons les hypothèses suivantes :

- transferts purement conductifs dans le soussol ;

— sous-sol thermiquement homogène et isotrope ;

- sous-sol infini dans toutes les directions, et limité intérieurement par un cylindre de longueur également infinie ;

- l'intérieur du puits (échangeur) est à une température imposée $T_{\mathrm{a}}$;

— la condition de flux à l'interface puits-sol est de type Fourier.

\section{Résolution par la méthode asymptotique.}

Le système d'équation de départ s'écrit :

$$
\begin{aligned}
& \frac{\partial^{2} T}{\partial r^{2}}+\frac{1}{r} \cdot \frac{\partial T}{\partial r}-\frac{1}{a} \cdot \frac{\partial T}{\partial t}=0 \\
& r=R_{\mathrm{p}} \Rightarrow-\left.\lambda \cdot \frac{\partial T}{\partial r}\right|_{r=R_{\mathrm{p}}}=k_{\mathrm{p}} \cdot\left(T_{\mathrm{a}}-T_{\mathrm{p}}\right) \\
& \left.r \rightarrow \infty \Rightarrow \frac{\partial T}{\partial r}\right|_{\infty} \rightarrow 0 \\
& t=0 \Rightarrow T=0 .
\end{aligned}
$$

Ce système a été résolu dans l'espace de Laplace [3], ce qui donne :

$$
\begin{aligned}
\tilde{T}(p, r) & =\frac{k_{\mathrm{p}}^{\prime} \cdot T_{\mathrm{a}}}{p} \times \\
& \times \frac{K_{0}(\omega \cdot r)}{\omega \cdot R_{\mathrm{p}} \cdot K_{1}\left(\omega \cdot R_{\mathrm{p}}\right)+k_{\mathrm{p}}^{\prime} \cdot K_{0}\left(\omega \cdot R_{\mathrm{p}}\right)} .
\end{aligned}
$$

Le problème qui se pose maintenant est de revenir à l'espace réel. La méthode générale est le calcul de l'intégrale complexe de Mellin-Fourier :

$$
T(t, r)=\frac{1}{2 \cdot i \cdot \pi} \cdot \int_{\gamma-i, \infty}^{\gamma+i \cdot \infty} \mathrm{e}^{p \cdot t} \cdot \tilde{T}(p, r) \cdot \mathrm{d} p .
$$

Cette intégrale se calcule généralement en utilisant le théorème des résidus sur le contour de Cauchy, composé du segment infini $\mathrm{AB}$ et de l'arc de cercle de rayon également infini $\mathrm{AA}^{\prime} \mathrm{B}^{\prime} \mathrm{B}$ (Fig. 1). Dans

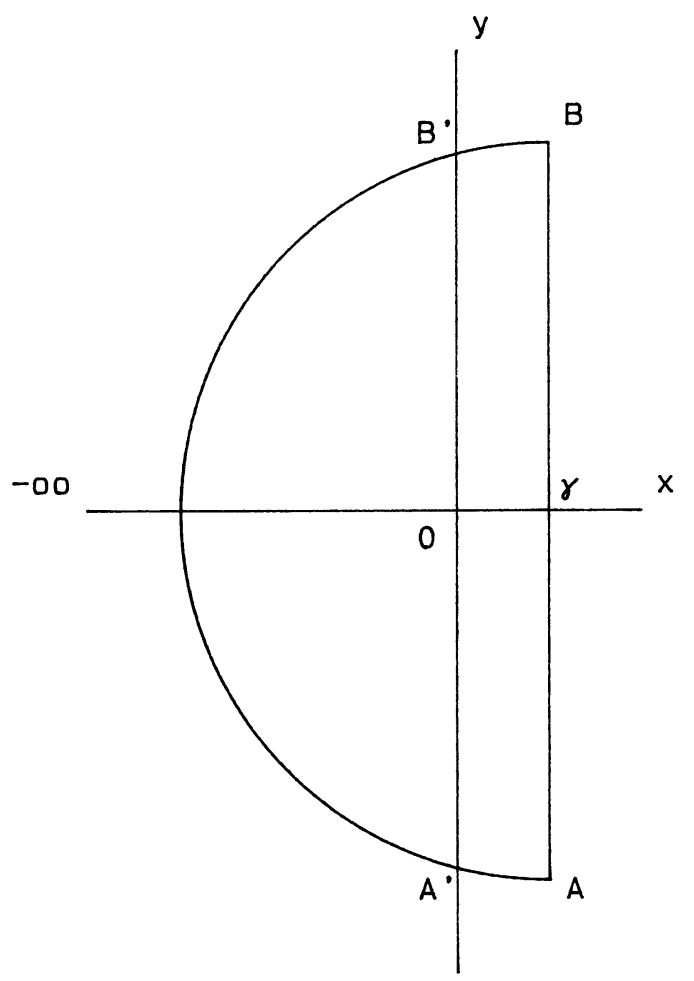

Fig. 1. - Contour de Cauchy.

[Cauchy's contour.]

notre cas il est possible de simplifier considérablement cette intégrale; en effet, notre fonction à intégrer ne comporte qu'une singularité, à l'origine, ce qui permet d'utiliser un contour modifié où nous contournons cette singularité (Fig. 2); ce contour est alors formé du même segment $\mathrm{AB}$, mais aussi des deux arcs de cercle, de rayons infinis, $\mathrm{AA}^{\prime} \mathrm{C}$ et $\mathrm{FB}^{\prime} \mathrm{B}$, d'un cercle de rayon infiniment petit $\mathrm{DE}$, et 


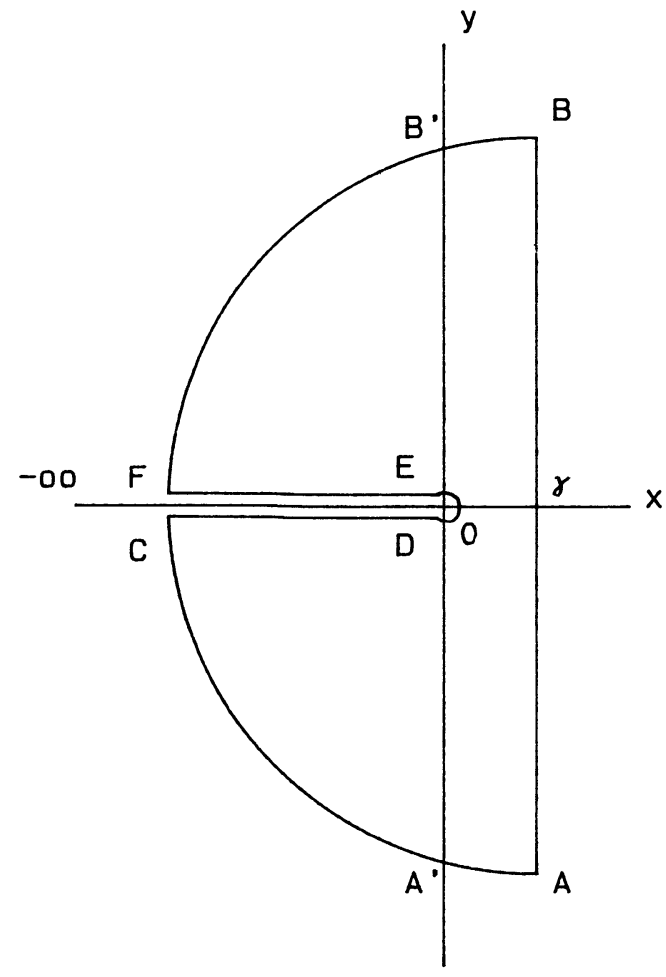

Fig. 2. - Contour modifié.

[Modified contour.] de deux segments réels $\mathrm{CD}$ et EF. Cette procédure nous permet alors de simplifier l'intégrale et d'écrire [3]

$$
T(t, r)=\frac{1}{2 \cdot i \cdot \pi} \cdot \int_{-\infty}^{0} \mathrm{e}^{p \cdot t} \cdot \tilde{T}(p \cdot r) \cdot \mathrm{d} p
$$

Nous savons [3,4] que dans la transformée de Laplace, aux grandes valeurs du temps correspondent les faibles valeurs de la variable de Laplace $p$. Or, dans le cas du stockage intersaisonnier, le chauffage dure relativement longtemps (quelques mois), ce qui rend une approximation aux temps longs tout à fait correcte. Nous pouvons donc utiliser une méthode asymptotique, qui consiste à remplacer la solution par son développement en série au voisinage de 0 . Pour cela, nous utilisons les développements limités des différentes fonctions élémentaires en tenant compte du fait que le rayon d'étude (quelques mètres) est grand devant le rayon du puits ; nous développons donc $K_{0}(\omega, r)$ au $4^{\mathrm{e}}$ ordre, alors que pour $K_{0}\left(\omega \cdot R_{\mathrm{p}}\right)$ et $K_{1}\left(\omega \cdot R_{\mathrm{p}}\right)$ nous nous contentons $\mathrm{du}$ premier ordre. Ce qui nous permet d'écrire, en posant $\alpha=\omega \cdot r$, et $\sum(1 / i)=$ $\sum_{i=1}^{n}\left(\frac{1}{i}\right):$

$$
\begin{aligned}
\frac{\tilde{T}(p \cdot r) \cdot p}{T_{\mathrm{a}} \cdot k_{\mathrm{p}}^{\prime}}= & \frac{K_{0}(\alpha)}{\omega \cdot R_{\mathrm{p}} \cdot K_{1}\left(\omega \cdot R_{\mathrm{p}}\right)+k_{\mathrm{p}}^{\prime} \cdot K_{0}\left(\omega \cdot R_{\mathrm{p}}\right)} \\
= & \frac{-I_{0}(\alpha) \cdot \log \left(\frac{\sigma \cdot \alpha}{2}\right)+\frac{\alpha^{2}}{4}+\frac{3}{2} \cdot \frac{\alpha^{4}}{64}+\frac{11}{6} \cdot \frac{\alpha^{6}}{2304}+\cdots+\sum\left(\frac{1}{i}\right) \cdot \frac{\alpha^{2 \cdot n}}{(n !)^{2} \cdot 2^{2 \cdot n}}}{1-k_{\mathrm{p}}^{\prime} \cdot \log \left(\frac{\sigma \cdot \omega \cdot R_{\mathrm{p}}}{2}\right)} .
\end{aligned}
$$

Posons

$$
\begin{aligned}
& X=\log \left(\frac{\sigma \cdot \omega \cdot R_{\mathrm{p}}}{2}\right)-\frac{1}{k_{\mathrm{p}}^{\prime}} \quad \text { et } \quad Y=\log \left(\frac{r}{R_{\mathrm{p}}}\right)+\frac{1}{k_{\mathrm{p}}^{\prime}} \\
& \frac{p \cdot \tilde{T}(p, r)}{T_{\mathrm{a}}}=I_{0}(\alpha)+\left(Y+\frac{\alpha^{2}}{4} \cdot(Y-1)+\frac{\alpha^{4}}{64} \cdot\left(Y-\frac{3}{2}\right)+\frac{\alpha^{6}}{2304} \cdot\left(Y-\frac{11}{6}\right)+\ldots\right. \\
& \left.\cdots+\frac{\alpha^{2 \cdot n}}{(n !)^{2} \cdot 2^{2 \cdot n}} \cdot[Y-\Sigma(1 / i)]\right) \cdot X^{-1} \text {. }
\end{aligned}
$$

Ceci permet d'écrire l'originale $T(t, r)$, en rappelant (2) et (3) :

$$
\begin{aligned}
\frac{2 \cdot i \cdot \pi}{T_{\mathrm{a}}} \cdot T(t, r)= & k_{\mathrm{p}}^{\prime} \cdot \int_{-\infty}^{0_{+}} \mathrm{e}^{p \cdot t} \cdot \frac{K_{0}(\omega \cdot r)}{\omega \cdot R_{\mathrm{p}} \cdot K_{1}\left(\omega \cdot R_{\mathrm{p}}\right)+k_{\mathrm{p}}^{\prime} \cdot K_{0}\left(\omega \cdot R_{\mathrm{p}}\right)} \cdot \frac{\mathrm{d} p}{p} \\
= & \int_{-\infty}^{0_{+}} \mathrm{e}^{p \cdot t} \cdot \frac{\mathrm{d} p}{p}+\int_{-\infty}^{0_{+}} \mathrm{e}^{p \cdot t} \cdot\left(\frac{r^{2}}{4 \cdot a}+\frac{p \cdot r^{4}}{64 \cdot a^{2}}+\frac{p^{2} \cdot r^{6}}{2302 \cdot a^{3}}+\cdots\right) \cdot \mathrm{d} p \\
& +Y \cdot \int_{-\infty}^{0_{+}} \mathrm{e}^{p \cdot t} \cdot X^{-1} \cdot \frac{\mathrm{d} p}{p}+\frac{Y-1}{4 \cdot a} \cdot r^{2} \cdot \int_{-\infty}^{0_{+}} \mathrm{e}^{p \cdot t} \cdot X^{-1} \cdot \mathrm{d} p \\
& +\frac{Y-3 / 2}{64 \cdot a^{2}} \cdot r^{4} \cdot \int_{-\infty}^{0_{+}} \mathrm{e}^{p \cdot t} \cdot X^{-1} \cdot p \cdot \mathrm{d} p \\
& +\frac{Y-11 / 6}{2304 \cdot a^{3}} \cdot r^{6} \cdot \int_{-\infty}^{0_{+}} \mathrm{e}^{p \cdot t} \cdot X^{-1} \cdot p^{2} \cdot \mathrm{d} p+\cdots
\end{aligned}
$$


dont le terme générique est :

$$
\begin{aligned}
\frac{Y-\sum(1 / i)}{(n !)^{2} \cdot 2^{2 \cdot n}} \cdot \frac{r^{2 \cdot n}}{a^{n}} \times & \\
& \times \int_{-\infty}^{0_{+}} \mathrm{e}^{p \cdot t} \cdot X^{-1} \cdot p^{n-1} \cdot \mathrm{d} p .
\end{aligned}
$$

Nous avons en outre pour les deux premiers termes [3] :

$$
\begin{gathered}
\frac{1}{2 \cdot i \cdot \pi} \cdot \int_{-\infty}^{0_{+}} \mathrm{e}^{p \cdot t} \cdot \frac{\mathrm{d} p}{p}=1 \\
\text { et } \frac{1}{2 \cdot i \cdot \pi} \cdot \int_{-\infty}^{0_{+}} \mathrm{e}^{p \cdot t} \cdot p^{n} \cdot \mathrm{d} p=0, n \in \mathbb{N} .
\end{gathered}
$$

Les autres intégrales sont de la forme suivante, établie par Ritchie et Sakakura [4] :

$$
I_{\nu}(v)=\frac{v^{-\nu}}{2 \cdot i \cdot \pi} \cdot \int_{-\infty}^{0_{+}} \frac{\mathrm{e}^{s} \cdot \mathrm{s}^{\nu-1} \cdot \mathrm{d} s}{(\log s-\log \nu)}
$$

avec $s=p \cdot t$ et $v=\frac{4 \cdot a \cdot t}{\sigma^{2} \cdot R_{\mathrm{p}}^{2}} \cdot \mathrm{e}^{2 / k_{\mathrm{p}}^{\prime}}$.

Soit :

$$
\begin{aligned}
& \frac{1}{2 \cdot i \cdot \pi} \cdot \int_{-\infty}^{0_{+}} \mathrm{e}^{p \cdot t} \cdot X^{-1} \cdot \frac{\mathrm{d} p}{p}=I_{0}(v) \\
& \frac{1}{2 \cdot i \cdot \pi} \cdot \int_{-\infty}^{0_{+}} \mathrm{e}^{p \cdot t} \cdot X^{-1} \cdot \mathrm{d} p=v \cdot I_{1}(v) \\
& \frac{1}{2 \cdot i \cdot \pi} \cdot \int_{-\infty}^{0_{+}} \mathrm{e}^{p \cdot t} \cdot X^{-1} \cdot p \cdot \mathrm{d} p=v^{2} \cdot I_{2}(v) \\
& \frac{1}{2 \cdot i \cdot \pi} \cdot \int_{-\infty}^{0_{+}} \mathrm{e}^{p \cdot t} \cdot X^{-1} \cdot p^{2} \cdot \mathrm{d} p=v^{3} \cdot I_{3}(v) \\
& \frac{1}{2 \cdot i \cdot \pi} \cdot \int_{-\infty}^{0_{+}} \mathrm{e}^{p \cdot t} \cdot X^{-1} \cdot p^{n-1} \cdot \mathrm{d} p=v^{n} \cdot I_{n}(v) .
\end{aligned}
$$

Ainsi, la solution du champ de température dans l'espace réel est :

$$
\begin{aligned}
& \frac{T(t, r)}{T_{\mathrm{a}}}=1+2 \cdot Y \cdot I_{0}(v)+ \\
& \quad+2 \cdot(Y-1) \cdot E \cdot I_{1}(v) \\
& \quad+\frac{Y-3 / 2}{2} \cdot E^{2} \cdot I_{2}(v)+\frac{Y-11 / 6}{18} \cdot E^{3} \cdot I_{3}(v)+\cdots \\
& \quad+2 \cdot \frac{Y-\sum(1 / i)}{(n !)^{2} \cdot 2^{2 \cdot n}} \cdot E^{n} \cdot I_{n}(v)
\end{aligned}
$$

avec $E=\frac{r^{2} \cdot \mathrm{e}^{2 / k_{\mathrm{p}}^{\prime}}}{R_{\mathrm{p}}^{2} \cdot \sigma^{2}}$

Cette expression est valable pour des temps relativement importants, mais il est à noter que le nombre de termes nécessaires à une bonne précision dépend du rayon d'étude, c'est-à-dire que pour étudier l'évolution de la température assez loin du puits il suffit de développer cette solution en utilisant le terme générique (5). Les intégrales (6) sont calculables par la relation [4] :

$$
I_{\nu}(v)=-\frac{(\log v)^{-1}}{v^{\nu}} \cdot \sum_{j=0}^{N} B_{j}^{\nu} \cdot(\log v)^{-j} .
$$

Les $B_{j}^{\nu}$ ont été tabulés par Ritchie et Sakakura [4] jusqu'à $j=5$; les valeurs du temps qui nous intéressent sont relativement importantes, ce qui fait que $v$ a un ordre de grandeur d'environ 4000 pour lequel la sommation précédente converge assez rapidement ; ainsi le $4^{\mathrm{e}}$ terme est 100 fois plus petit que le premier ou le second.

Avec la relation (6) nous avons obtenu une expression analytique du champ de température dans le sol à tout instant pour des temps longs. Cette forme présente un double avantage : d'une part elle est calculable beaucoup plus rapidement que par des méthodes purement numériques, et, d'autre part, il est possible de la simplifier encore davantage en limitant le nombre de termes de la série dans le cas où seul un ordre de grandeur nous intéresse.

Pour apprécier la validité de cette méthode de calcul, nous la comparons à deux autres méthodes d'inversion :

- La première, méthode intégrale, consiste à inverser analytiquement l'intégrale de Mellin-Fourier en intégrale réelle, puis à intégrer celle-ci numériquement par l'algorithme de Gauss.

- La seconde, qui est une méthode d'inversion numérique, a été développée par Gaver [6].

Examinons tout d'abord chacune de ces méthodes ; un exemple numérique de comparaison sera ensuite présenté au paragraphe 4 .

\section{Méthode intégrale.}

2.1 INVERSION. - Nous rappelons la solution de Mellin-Fourier (3) :

$$
\begin{aligned}
T(t, r) & =\frac{k_{\mathrm{p}}^{\prime} \cdot T_{\mathrm{a}}}{2 \cdot i \cdot \pi} \cdot \int_{\gamma-i \cdot \infty}^{\gamma+i \cdot \infty} \mathrm{e}^{p \cdot t} \times \\
& \times \frac{K_{0}(\omega \cdot r)}{\omega \cdot R_{\mathrm{p}} \cdot K_{1}\left(\omega \cdot R_{\mathrm{p}}\right)+k_{\mathrm{p}}^{\prime} \cdot K_{0}\left(\omega \cdot R_{\mathrm{p}}\right)} \cdot \frac{\mathrm{d} p}{p} .
\end{aligned}
$$

Pour inverser cette intégrale complexe en intégrale réelle, nous reprenons le contour modifié (Fig. 2) ; et nous décomposons tronçon par tronçon, ce qui donne :

- Une intégrale sur le cercle DE qui tend vers $\frac{2 \cdot i \cdot \pi}{k_{\mathrm{p}}^{\prime}}$. 
- Deux intégrales, sur CD et EF :

$$
\begin{aligned}
T(t, r) & =\frac{1}{2 \cdot i \cdot \pi} \times \int_{\mathrm{CD}} \mathrm{e}^{p \cdot t} \cdot \tilde{T}(p, r) \cdot \mathrm{d} p+ \\
& +\frac{1}{2 \cdot i \cdot \pi} \times \int_{\mathrm{EF}} \mathrm{e}^{p \cdot t} \cdot \tilde{T}(p, r) \cdot \mathrm{d} p+T_{\mathrm{a}}
\end{aligned}
$$

Nous effectuons alors les changements de variables :

$$
\begin{array}{ll}
p=a \cdot u^{2} \cdot \mathrm{e}^{-i \cdot \pi} & \text { sur le tronçon CD } \\
p=a \cdot u^{2} \cdot \mathrm{e}^{i \cdot \pi} & \text { sur le tronçon EF }
\end{array}
$$

et, sachant que les fonctions de Bessel à variable complexe s'écrivent :

$$
\begin{aligned}
K_{0}(i \cdot x) & =\frac{\pi \cdot i}{2} \cdot\left(J_{1}(x)+i \cdot Y_{0}(x)\right) \\
K_{1}(i \cdot x) & =\frac{-\pi \cdot i}{2} \cdot\left(J_{0}(x)+i \cdot Y_{0}(x)\right) \\
K_{0}(-i \cdot x) & =\frac{-\pi \cdot i}{2} \cdot\left(J_{0}(x)-i \cdot Y_{0}(x)\right) \\
K_{1}(-i \cdot x) & =\frac{-\pi}{2} \cdot\left(J_{0}(x)-i \cdot Y_{1}(x)\right)
\end{aligned}
$$

nous obtenons, après addition de toutes les portions du contour :

$$
\begin{array}{cc}
T(t, r)=T_{\mathrm{a}}+2 \cdot k_{\mathrm{p}}^{\prime} \cdot T_{\mathrm{a}} \cdot \int_{0}^{+\infty} \mathrm{e}^{-t^{\prime} \cdot u^{2}} \times \\
& \times \frac{J_{0}\left(u \cdot r^{\prime}\right) \cdot Y(u)-Y_{0}\left(u \cdot r^{\prime}\right) \cdot J(u)}{(J(u))^{2}+(Y(u))^{2}} \cdot \frac{\mathrm{d} u}{u} \\
\text { où } & J(u)=u \cdot J_{1}(u)+k_{\mathrm{p}}^{\prime} \cdot J_{0}(u) \\
\text { et } & Y(u)=u \cdot Y_{1}(u)+k_{\mathrm{p}}^{\prime} \cdot Y_{0}(u) .
\end{array}
$$

Ceci nous donne une solution analytique sous la forme d'une intégrale réelle, laquelle n'est pas calculable par les méthodes classiques. Nous utiliserons donc le calcul numérique. Pour appliquer un algorithme de calcul à une intégrale, il faut que celleci obéisse à un certain nombre de critères parmi lesquels :

- avoir un domaine d'intégration fini ;

- ne pas avoir de point singulier sur ce domaine.

Or, l'intégrale (8), dans notre cas, ne répond à aucun de ces deux critères puisque la borne supérieure est infinie, et que la fonction tend vers $-\infty$ quand $u$ tend vers 0 . Le tracé de la fonction (Fig. 3) permet de constater, cependant, qu'elle

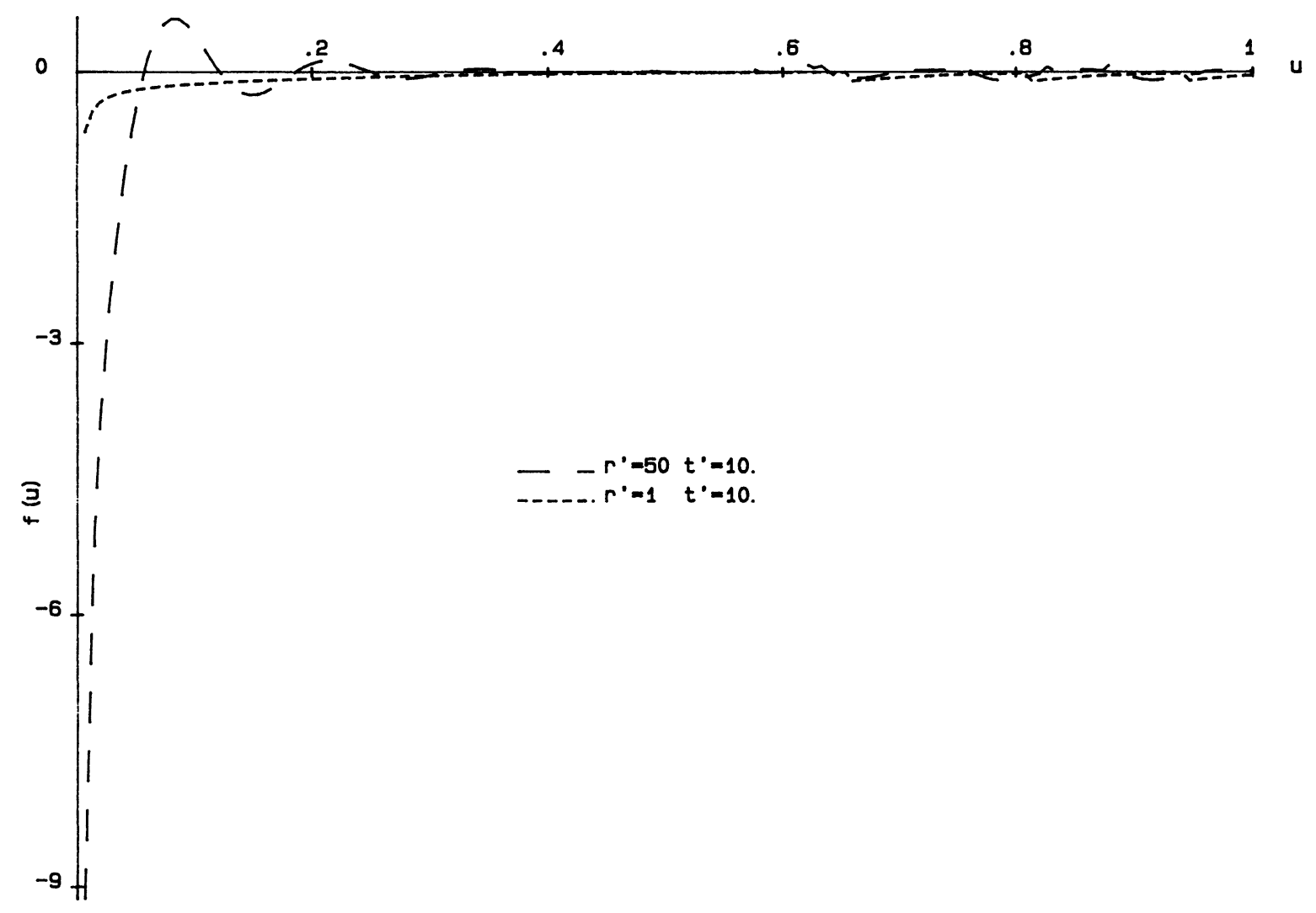

Fig. 3. - Variation de l'intégrande.

[Variation of the integrand.] 
tend rapidement vers 0 , ce qui nous amène à adopter une solution proposée par Germant [5] ; on procède au tronçonnage du domaine d'intégration en trois sous-domaines :

$$
\int_{0}^{\infty}=\int_{0}^{\varepsilon_{1}}+\int_{\varepsilon_{1}}^{\varepsilon_{2}}+\int_{\varepsilon_{2}}^{\infty}
$$

chacune de ces intégrales ayant pour caractéristique : $\int_{0}^{\varepsilon_{1}}$ : intégrable analytiquement après développement en série.

$\int_{\varepsilon_{1}}^{\varepsilon_{2}}$ : intégrable numériquement.

$\int_{\varepsilon_{2}}^{\infty}$ : tend vers une valeur nulle.

Ce procédé est valable pour toute intégrale convergente dont l'intégrande est développable en série au voisinage de ses singularités, à condition que ce développement soit intégrable analytiquement.
2.2 Calcul de l'intégrale $\int_{0}^{\varepsilon_{1}}$. - Pour la première intégrale, le choix se portera sur une valeur suffisamment petite, de façon à pouvoir remplacer les différentes fonctions élémentaires qui apparaissent dans (8) par leurs développements en série classiques au voisinage de 0 . Les trois conditions à remplir pour approximer les fonctions par leurs développements au premier ordre sont :

$$
t^{\prime} \cdot u^{2}<0,01 ; \quad r^{\prime} \cdot u<0,1 ; u<0,1 \text {. }
$$

Dans notre cas, la valeur maximale de $t^{\prime}$ est de l'ordre de 1000 (ce qui correspond à une durée de chauffage d'environ 5 mois), et le rayon d'étude est à peu près de $4 \mathrm{~m}$ pour un rayon de puits de $0,08 \mathrm{~m}$, la valeur maximale de $r^{\prime}$ est donc de 50. D'où la condition suffisante : $u<0,001$ qui est la valeur maximale admissible pour $u$; nous posons alors $\varepsilon_{1}=0,001$.

L'utilisation des développements nous permet d'écrire :

$$
\begin{aligned}
f(u) & =\mathrm{e}^{-t^{\prime} \cdot u^{2}} \cdot \frac{J_{0}\left(u \cdot r^{\prime}\right) \cdot Y(u)-Y_{0}\left(u \cdot r^{\prime}\right) \cdot J(u)}{(J(u))^{2}+(Y(u))^{2}} \cdot \frac{1}{u} \\
& =-\frac{\pi}{2} \cdot \frac{k_{\mathrm{p}}^{\prime} \cdot \log r^{\prime}+1}{\left(\pi \cdot k_{\mathrm{p}}^{\prime} / 2\right)^{2}+\left(k_{\mathrm{p}}^{\prime} \cdot \log (\sigma \cdot u / 2)-1\right)^{2}} \cdot \frac{1}{u} .
\end{aligned}
$$

Nous illustrons la validité de cette approximation dans le cas le plus défavorable, c'est-à-dire pour $r^{\prime}=50$ (Fig. 4). En outre, nous constatons que pour $u$ petit, $f(u)$ ne dépend plus que du rayon. L'expression (9) est analytiquement intégrable, ce qui, en passant par le changement de variable :

$$
U=\frac{2}{\pi} \cdot\left(\log \left(\frac{\sigma \cdot u}{2}\right)-\frac{1}{k_{\mathrm{p}}^{\prime}}\right)
$$

donne l'écriture de l'intégrale :

$$
\begin{aligned}
& \int_{0}^{\varepsilon_{1}} f(u) \cdot \mathrm{d} u=\frac{1+k_{\mathrm{p}}^{\prime} \cdot \log r^{\prime}}{k_{\mathrm{p}}^{\prime}} \times\left(-\frac{\pi}{2}\right) \times \\
& \quad \times\left\{\operatorname{arctg}\left[\frac{2}{\pi} \cdot\left(\frac{1}{k_{\mathrm{p}}^{\prime}}-\log \left(\frac{\sigma \cdot \varepsilon_{1}}{2}\right)\right)\right]-\frac{\pi}{2}\right\} .
\end{aligned}
$$

2.3 CalCul de l'intégrale $\int_{\varepsilon_{1}}^{\varepsilon_{2}}$. - Pour le calcul numérique de la seconde intégrale, nous utilisons l'algorithme de Gauss. Celui-ci fait appel à une base de polynômes de Legendre sur laquelle on développe la fonction à intégrer, puis on écrit que l'intégrale est égale à la somme des racines de ces polynômes pondérées par un certain coefficient. Les polynômes de Legendre étant définis sur l'intervalle $[-1,+1]$, il nous faut procéder au changement de variable :

$$
\begin{aligned}
x_{k}=\frac{b+a}{2}+\frac{b-a}{2} \cdot \zeta_{k} \text { où } \quad x_{k} \in[a, b] \\
\text { et } \zeta_{k} \in[-1,+1]
\end{aligned}
$$

d'où la valeur de l'intégrale :

$$
\int_{a}^{b} g(x) \cdot \mathrm{d} x=\frac{b-a}{2} \cdot \sum_{k=1}^{N} \delta_{k} \cdot g\left(x_{k}\right) .
$$

Les $\delta_{k}$ et $\zeta_{k}$ sont tabulés [7] et dépendent de la valeur de $n$ choisie, laquelle correspond au degré des polynômes par lesquels on approxime la fonction. Si nous prenons $n=12$, c'est-à-dire des polynômes du $12^{c}$ degré, en tenant compte que les racines sont symétriques par rapport à l'origine, nous écrivons :

$$
\int_{\varepsilon_{2}}^{\varepsilon_{1}} f(u) \cdot \mathrm{d} u=\frac{\varepsilon_{2}-\varepsilon_{1}}{2} \times \sum_{k=1}^{12} \delta_{k} \cdot\left[f\left(x_{k}\right)\right] .
$$

La détermination de $\varepsilon_{2}$ se fait empiriquement par appréciation de la valeur minimale de $u$ à partir de 


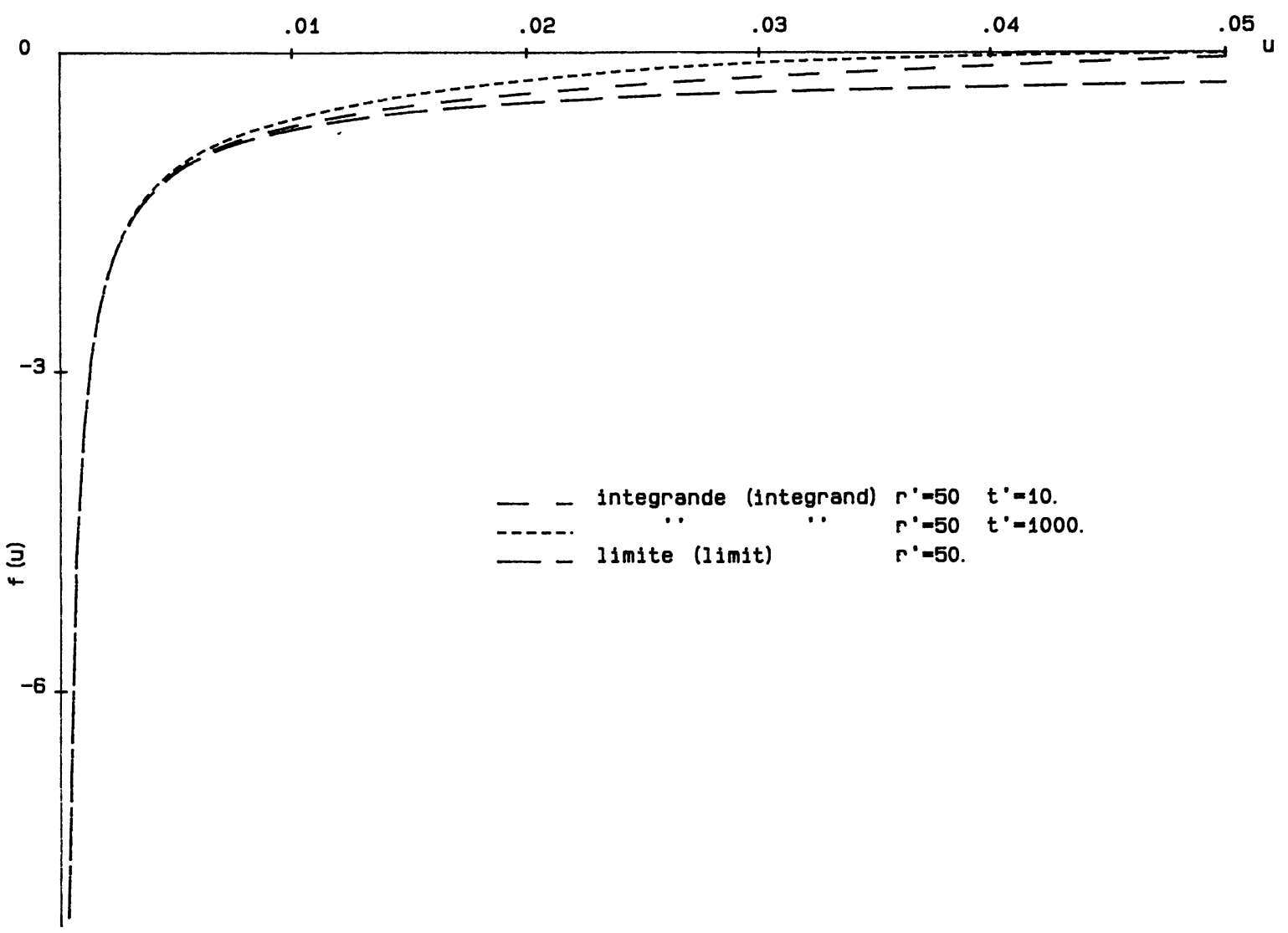

Fig. 4. - Variation de l'intégrande et de sa limite.

[Variation of the integrand and its limit.]

laquelle $f(u)$ devient quasiment nulle (Fig. 3) ; nous constatons que déjà pour $u=1, f(u)$ est très petit, aussi pour conserver une marge de sécurité nous prenons $\varepsilon_{2}=10$.

2.4 INTÉGRALE $\int_{\varepsilon_{2}}^{+\infty} \cdot-$ Nous rappelons (Sect. 2.1) que d'après notre méthode de calcul :

$$
\int_{\varepsilon_{2}}^{+\infty} f(u) \cdot \mathrm{d} u=0
$$

Nous obtenons alors la température en tout point du sol par addition des deux résultats sur $\left[0, \varepsilon_{1}\right]$ et $\left[\varepsilon_{1}, \varepsilon_{2}\right]$.

\section{Méthode de Gaver.}

La seconde méthode qui nous servira d'élément de comparaison est une méthode d'inversion numérique développée par Gaver [6] où la valeur de la fonction en un point est calculée par la relation suivante :

$$
g(t)=\frac{\log 2}{t} \cdot \sum_{j=1}^{N} V_{j} \cdot \tilde{g}\left(\frac{j \cdot \log 2}{t}\right)
$$

$$
\begin{aligned}
V_{j}= & \sum_{k=E\left[\frac{j+1}{2}\right]}^{\min (j, N / 2)} \times \\
& \times \frac{k^{N / 2} \cdot(2 . k) ! \cdot(-1)^{j-N / 2}}{(N / 2-k) ! \cdot(k-1) ! \cdot k ! \cdot(j-k) ! \cdot(2 . k-j) !} \cdot
\end{aligned}
$$

Le choix de la valeur de $N$ se fait à partir de la précision disponible sur la machine de calcul; ainsi pour une précision de 8 chiffres on prend $N=10$. Ce qui, appliqué dans notre cas nous donne :

$$
\begin{aligned}
& T(t, r)=k_{\mathrm{p}}^{\prime} \cdot T_{\mathrm{a}} \cdot \sum_{j=1}^{10} \frac{V_{j}}{j} \times \\
& \left.\quad \times \frac{K_{0}\left(\omega_{j} \cdot r\right)}{\omega_{j} \cdot R_{\mathrm{p}} \cdot K_{1}\left(\omega_{j} \cdot R_{\mathrm{p}}\right)+k_{\mathrm{p}}^{\prime} \cdot K_{0}\left(\omega_{j} \cdot R_{\mathrm{p}}\right.}\right)
\end{aligned}
$$

avec

$$
\omega_{j}=\sqrt{\frac{j \cdot \log 2}{a \cdot t}}
$$

Le calcul se fait donc par une sommation que nous avons programmée sur micro-ordinateur. $\mathrm{Au}$ vu de l'expression donnée par Gaver, apparaît la possibilité de considérer la solution (11) comme un résultat analytique que nous exploiterons par la suite. 


\section{Comparaison des trois méthodes.}

Afin de vérifier l'équivalence de ces trois méthodes, nous avons tracé les courbes représentant le champ de température dans le sol, donné par chacune d'elles. En abscisse nous avons $r^{\prime}$ (rayon adimensionnel) et en ordonnée $T^{\prime}=T / T_{\mathrm{a}}$ (température adimensionnelle). Les calculs sont effectués à différents instants $t^{\prime}$ de la phase de chauffage du sol.

Nous avons dessiné trois graphiques correspondants à $t^{\prime}=250,500$ et 1000 , pour chacun desquels $r^{\prime}$ varie de 1 à 100 ; la valeur du coefficient global d'échange adimensionnel a été fixée à 2 .

Nous avons les différents champs de température (Figs. 5, 6, 7) obtenus par ces trois méthodes d'inversion pour les trois instants de calcul. Nous constatons en premier lieu une coïncidence parfaite entre la méthode intégrale et la méthode de Gaver, au moins jusqu'à un rayon $r^{\prime}=100$. Ensuite, nous observons une très bonne concordance de la méthode asymptotique avec les deux autres, dites « exactes », jusqu'à un certain rayon limite, qui augmente avec la durée de chauffage. L'existence de cette limite était prévisible du fait que cette méthode s'applique aux temps longs et aux rayons pas trop grands. Ainsi, le rayon limite passe de $r^{\prime}=40$ pour $t^{\prime}=250$, à $r^{\prime}=60$ pour $t=500$ et à $r^{\prime}=80$ pour $t^{\prime}=1000$. Pour que la méthode reste valable avec des rayons plus importants, il suffit de développer la solution proposée à un ordre supérieur grâce au terme générique (7).
La pratique nous amène à faire quelques remarques sur ces modes de résolution :

La méthode asymptotique est de loin la plus rapide en temps de calcul et la plus facile à programmer; cependant elle présente l'inconvénient de n'être utilisable que pour des temps adimensionnels relativement importants, c'est-à-dire des temps longs et un rayon de puits pas trop grand.

La méthode de Gaver est aussi relativement simple à programmer mais demande des calculs plus importants (fonctions de Bessel) et elle est donc plus longue ; mais elle est toujours applicable.

La méthode intégrale est, quant à elle, la moins intéressante des trois car si elle a la même validité que la numérique elle est compliquée en programmation et demande des temps de calcul très importants.

\section{Expérimentation.}

5.1 DESCRIPTION. - Le calage du modèle précédemment décrit se fait sur une installation expérimentale composée d'un échangeur baïonnette vertical de $25 \mathrm{~m}$ de long, ayant pour diamètre extérieur $0,16 \mathrm{~m}$. En surface, nous avons raccordé un réchauffeur électrique, d'une puissance de $3 \mathrm{~kW}$, et un accélérateur de circulation. Un compteur d'eau permet de mesurer le débit moyen d'eau passant dans le puits, et une batterie de 25 couples thermoélectriques de type $\mathrm{K}$ (Chromel-Alumel) sont dissé-

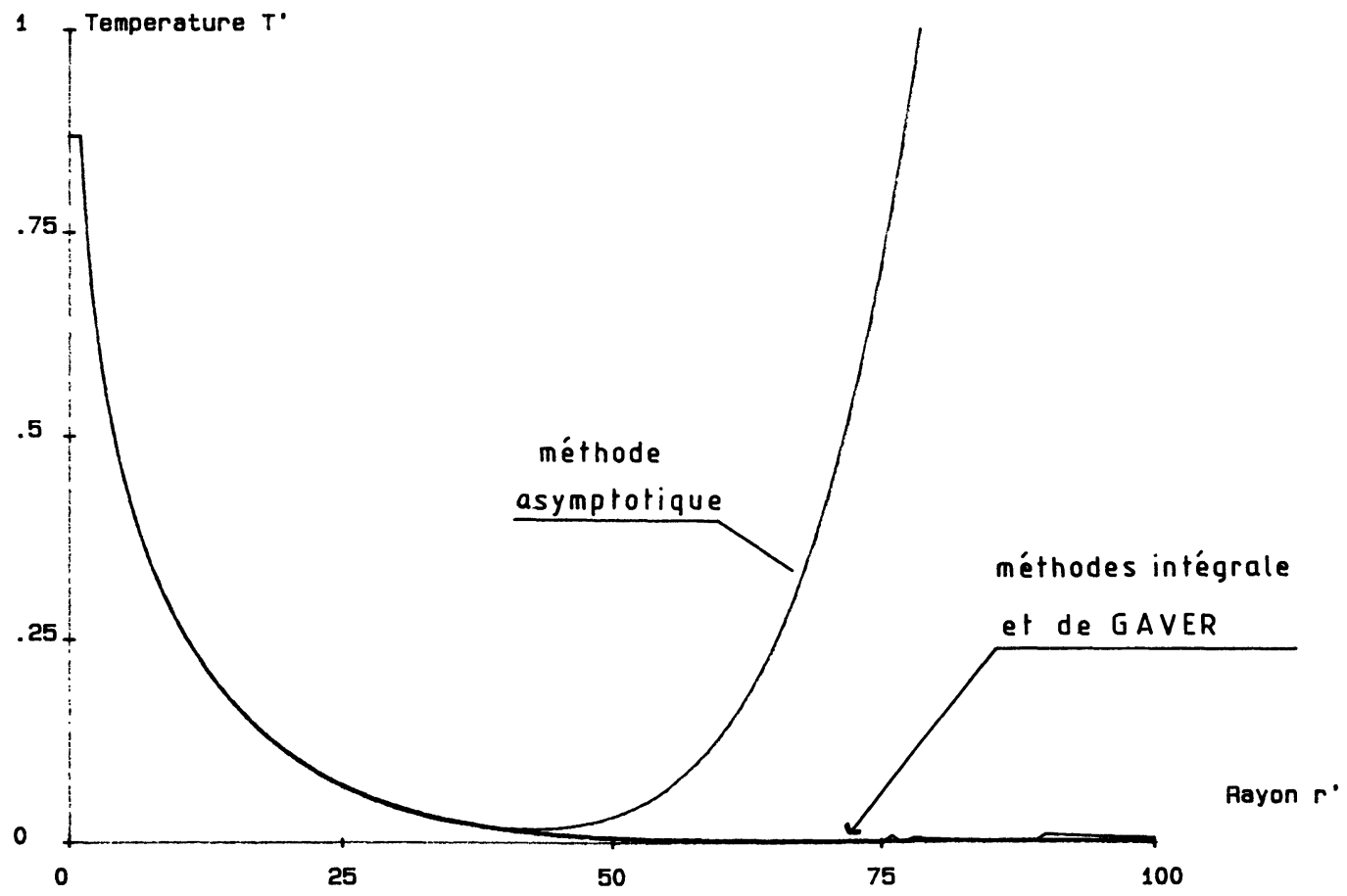

Fig. 5. - Température adimensionnelle du sol en fonction de $r^{\prime}$ par les trois méthodes de calcul pour $t^{\prime}=250$.

[Adimensionnal ground temperature function of $r^{\prime}$ by the three methods of calculation for $t^{\prime}=250$.] 


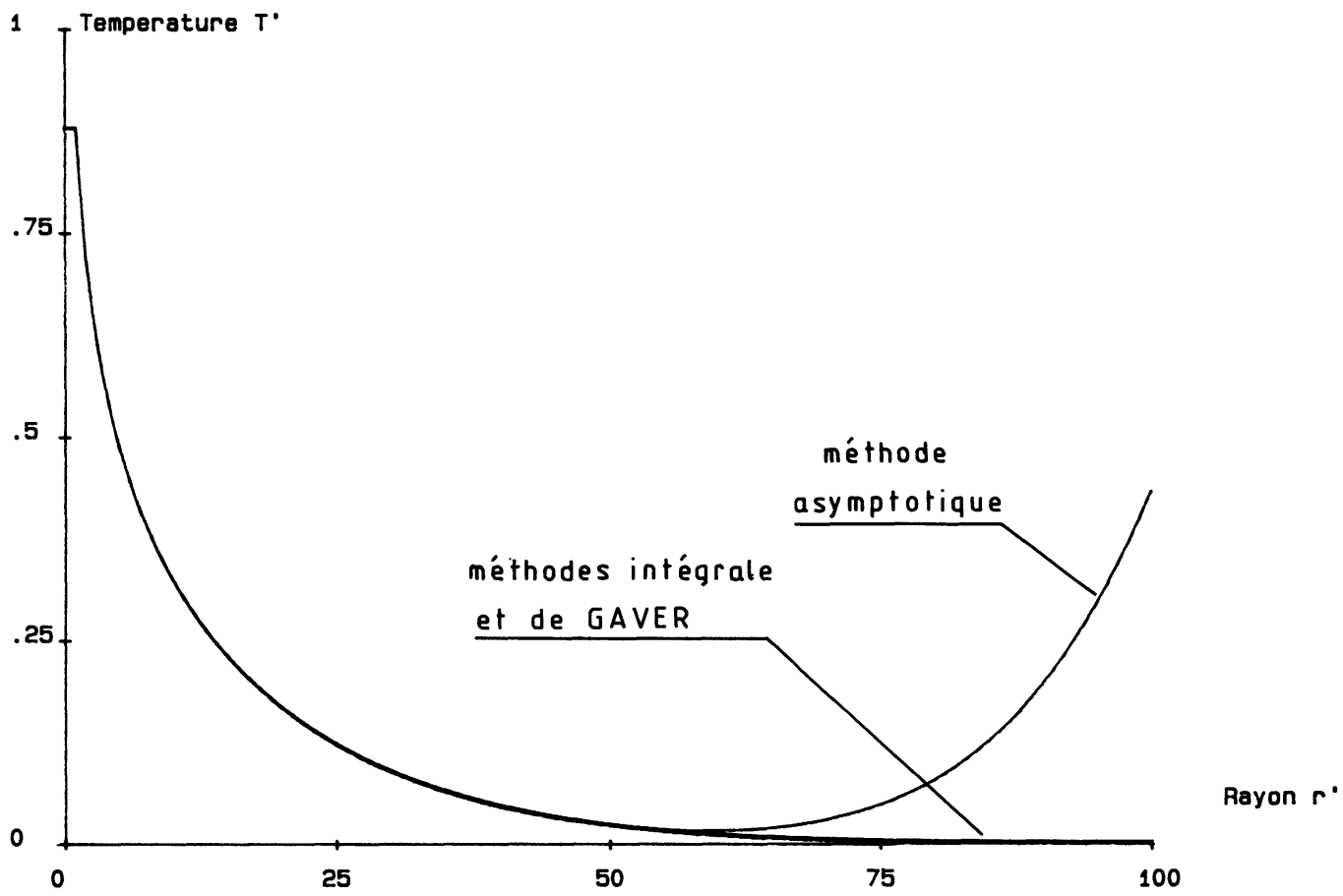

Fig. 6. - Idem avec $t^{\prime}=500$.

[Idem with $t^{\prime}=500$.]

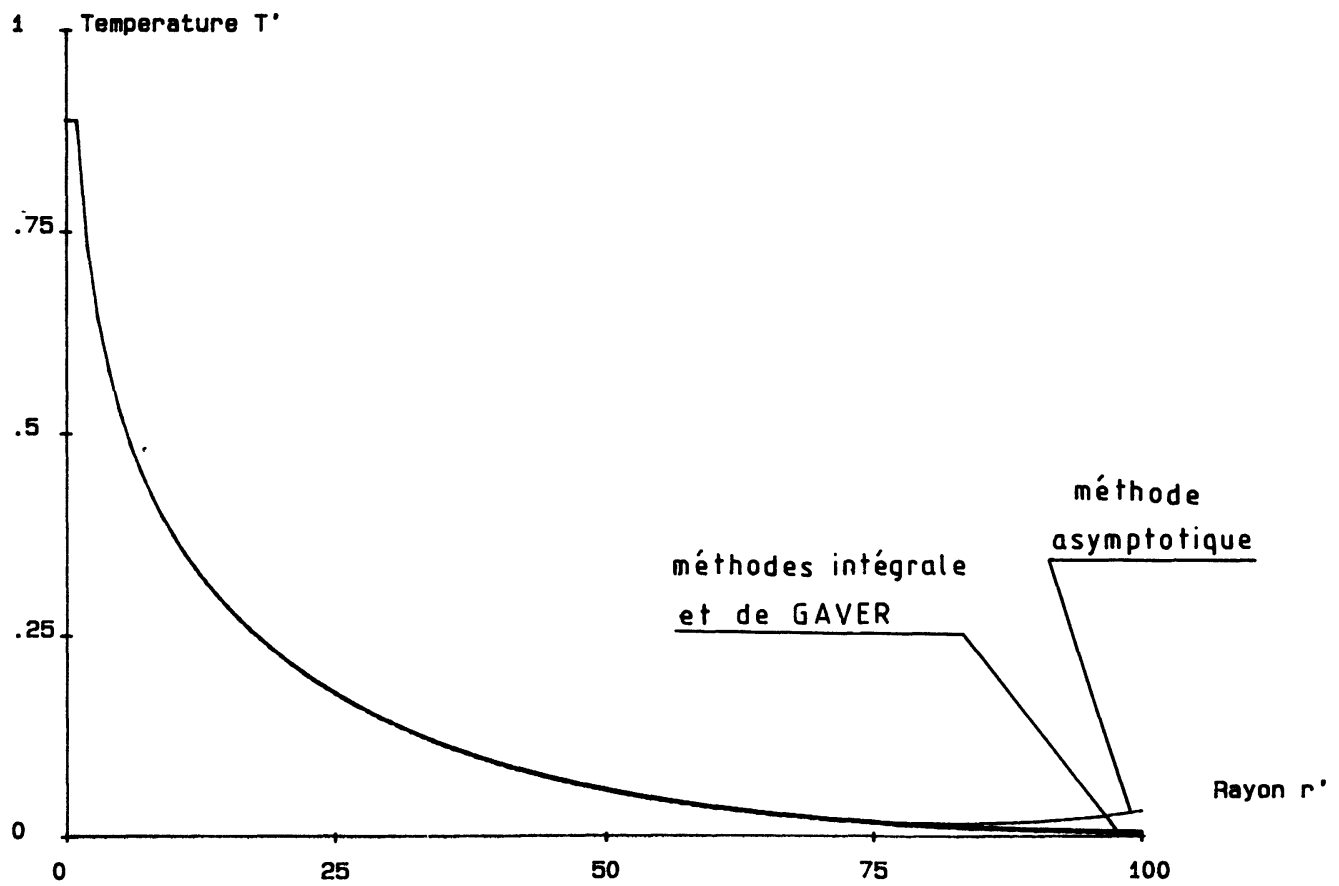

Fig. 7. - Idem avec $t^{\prime}=1000$.

[Idem with $t^{\prime}=1000$.]

minés à l'intérieur de l'échangeur et dans le sol (Fig. 8), à proximité du puits. Pour l'étude du comportement du milieu stockeur, nous ne retenons que 8 points de mesures de températures :

- les températures d'entrée et de sortie de l'eau afin de connaître la température moyenne dans le puits ;

- les 6 températures du sol mesurées suivant deux verticales, situées aux rayons $0,80 \mathrm{~m}$ et $1,20 \mathrm{~m}$ par rapport au forage principal. Sur ces axes, nous mesurons la température du sol aux profondeurs : $5 \mathrm{~m}, 10 \mathrm{~m}$ et $15 \mathrm{~m}$.

Afin de déterminer les paramètres thermophysiques de la craie, le BRGM a procédé à un carottage sur le site et à l'extraction d'un échantillon de craie dans une carrière distante d'une trentaine de kilomè- 


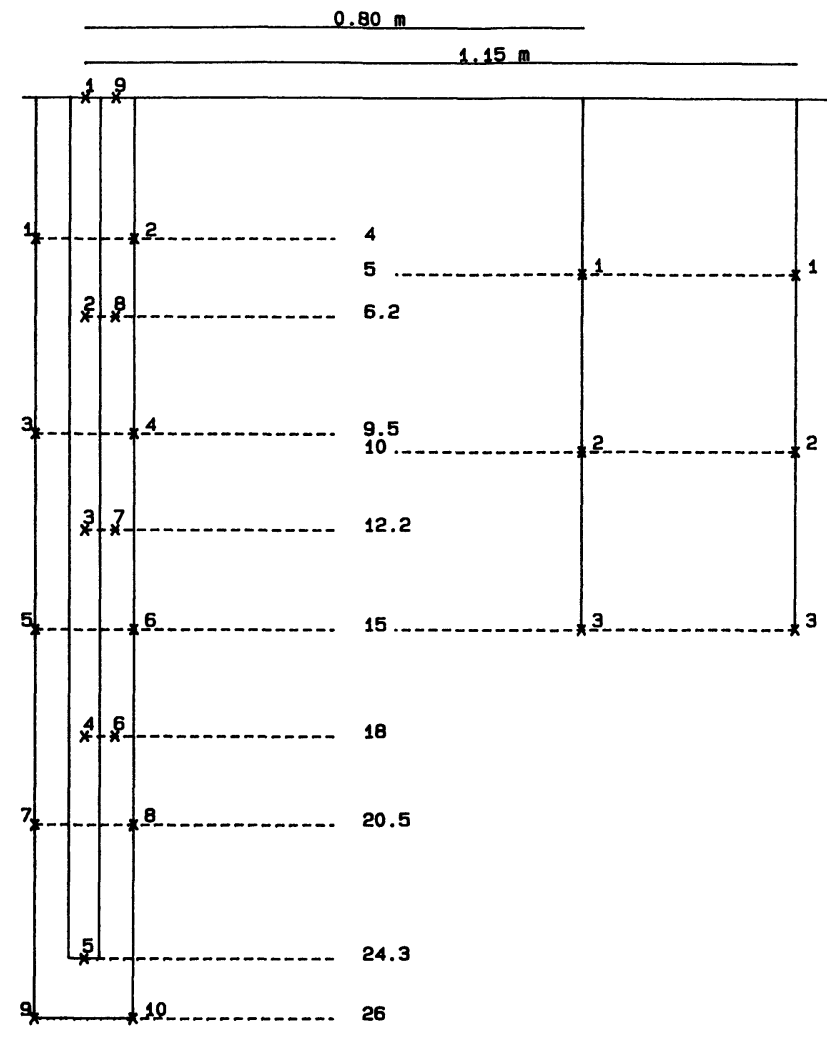

Fig. 8. - Disposition des thermocouples.

[Location of the thermocouples.]

tres. Les mesures de laboratoire faites, d'une part au BRGM d'Orléans sur les carottes pour la conductivité, et d'autre part à l'Ecole des Mines de Nancy sur l'échantillon sec pour la diffusivité, ont donné les valeurs suivantes : $\lambda=1,8 \mathrm{~W} / \mathrm{m} . \mathrm{K}$ et $a=$ $0,585 \times 10^{-6} \mathrm{~m}^{2} / \mathrm{s}$. L'absence de mesure in situ nous engage à la prudence quant aux valeurs numériques obtenues, aussi, nous ne considérons ces mesures que comme des ordres de grandeur.

Le coefficient global d'échange entre le fluide caloporteur et le sol a été estimé d'après une relation empirique concernant les espaces annulaires [9] :

$$
\begin{aligned}
N u=\left(\frac{D_{\mathrm{e}}}{D_{i}}\right)^{0,8} \cdot\left(\operatorname{Re} \cdot \operatorname{Pr} \cdot \frac{D_{\mathrm{h}}}{L}\right)^{0,45} \times & \\
& \times G r^{0,05}=\frac{h_{\mathrm{e}} \cdot D_{\mathrm{h}}}{\lambda_{\mathrm{f}}}
\end{aligned}
$$

avec $D_{\mathrm{e}}$ et $D_{i}$ : diamètres extérieur et intérieur, $D_{\mathrm{h}}$ : diamètre hydraulique, $L$ : longueur du tube, $h_{\mathrm{e}}$ : coefficient de convection sur la paroi externe et $\lambda_{\mathrm{f}}$ : conductivité du fluide; ce qui nous donne, en rajoutant la résistance thermique de la paroi, un coefficient global d'échange d'environ $k_{\mathrm{p}}=$ $70 \mathrm{~W} / \mathrm{m}^{2} .{ }^{\circ} \mathrm{C}$.

Une étude de sensibilité (Fig. 9) nous permet de constater que pour $k_{\mathrm{p}}<50 \mathrm{~W} / \mathrm{m}^{2}$.K le flux échangé augmente très rapidement, puis, sitôt que l'on atteint des valeurs de $k_{\mathrm{p}}>50 \mathrm{~W} / \mathrm{m}^{2} . \mathrm{K}$, celui-ci n'a plus aucune influence sur les échanges entre le puits et l'annulaire, et ce quelque soit la durée de chauffage prise en considération. Nous considérons donc que le fait de calculer ce coefficient d'échange par une méthode empirique même précise à 10 ou $20 \%$ est suffisant à condition que la valeur de ce dernier ne puisse pas être inférieure à $50 \mathrm{~W} / \mathrm{m}^{2} . \mathrm{K}$.

5.2 DÉroulement. - L'expérimentation s'est déroulée en trois temps: une première phase de stockage pendant deux mois, suivie d'une relaxation d'une durée de six mois pour revenir aux conditions initiales, et enfin, une seconde période de chauffage de un mois et demi cette fois-ci. Durant les deux manipulations nous avons commencé par un faible débit d'eau, pour augmenter plus rapidement la température à l'intérieur du puits, ensuite la stabilisation thermique s'est faite en portant le débit à sa valeur maximale, soit approximativement $1 \mathrm{~m}^{3} / \mathrm{h}$.

5.2.1 Manipulation I. - Cette première phase de stockage s'est déroulée pendant 1500 heures. La température d'entrée d'eau a été stabilisée à environ $55^{\circ} \mathrm{C}$ après 750 heures de fonctionnement. Les relevés de température du sol mettent d'abord en évidence un gradient vertical, orienté de bas en haut, de la température initiale. L'évolution au cours du temps du niveau thermique du sol se fait en conservant le même sens à ce gradient qui devient plus important à 1,20 m qu'à $0,80 \mathrm{~m}$ (Fig. 10).

5.2.2 Relaxation. - Cette phase de retour aux conditions initiales a duré 6 mois, pendant lesquels nous avons observé la descente en température. A proximité immédiate du puits, cette chute a été très rapide les premiers jours, puis de plus en plus lente. C'est-à-dire que s'est appauvrie en quelques jours seulement la zone de stockage où la chaleur était à un niveau thermique suffisant pour être récupérable. En fin de relaxation, le sol est presque revenu à sa température originelle, mais avec cependant une différence : les températures initiales à $10 \mathrm{~m}$ et $15 \mathrm{~m}$ sont devenues supérieures à la température initiale à $5 \mathrm{~m}$. Cette remarque semble s'expliquer par des hétérogénéités dans le sol donnant une diffusivité décroissante avec la profondeur. De plus, nous avons constaté, après coup, l'existence d'une faille contenant un matériau différent, laquelle pourrait être à l'origine d'une inertie thermique du sol plus grande en profondeur, d'où la présence de ce reliquat du stockage précédent à $10 \mathrm{~m}$ et $15 \mathrm{~m}$, et son absence à $5 \mathrm{~m}$.

5.2.3 Manipulation II. - Cette seconde phase de chauffage a duré environ 1200 heures, et nous avons procédé de la même manière que précédemment avec une température d'entrée d'eau stabilisée à 


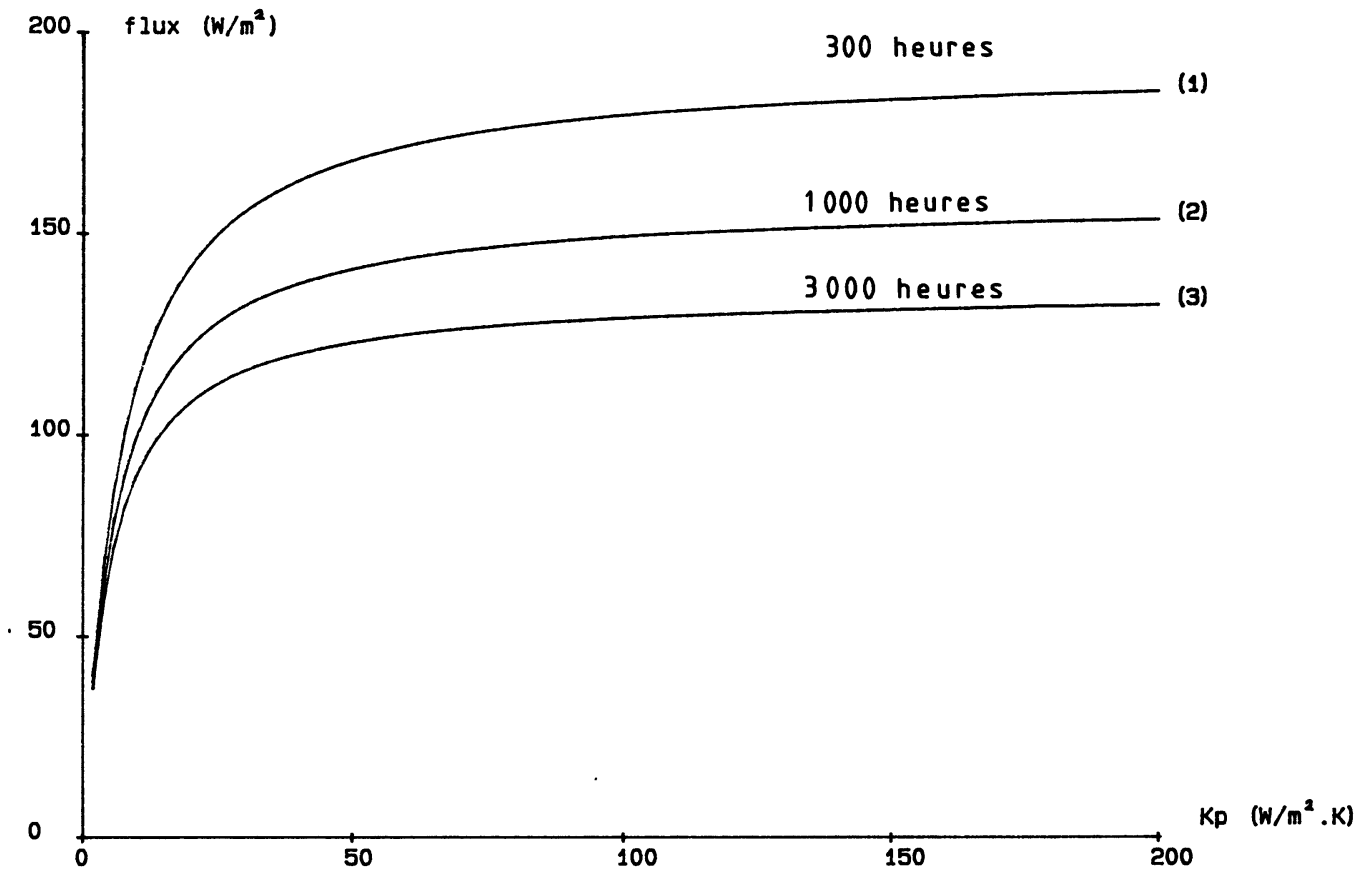

Fig. 9. - Sensibilité de la densité de flux à la paroi en fonction du coefficient d'échange pour $t=300 \mathrm{~h}(1), 1000 \mathrm{~h}(2)$, 3000 h (3).

[Sensibility of the wall rate density function of the exchange coefficient for $t=300 \mathrm{~h}(1), 1000 \mathrm{~h} \mathrm{(2),} 3000 \mathrm{~h}(3)$.]

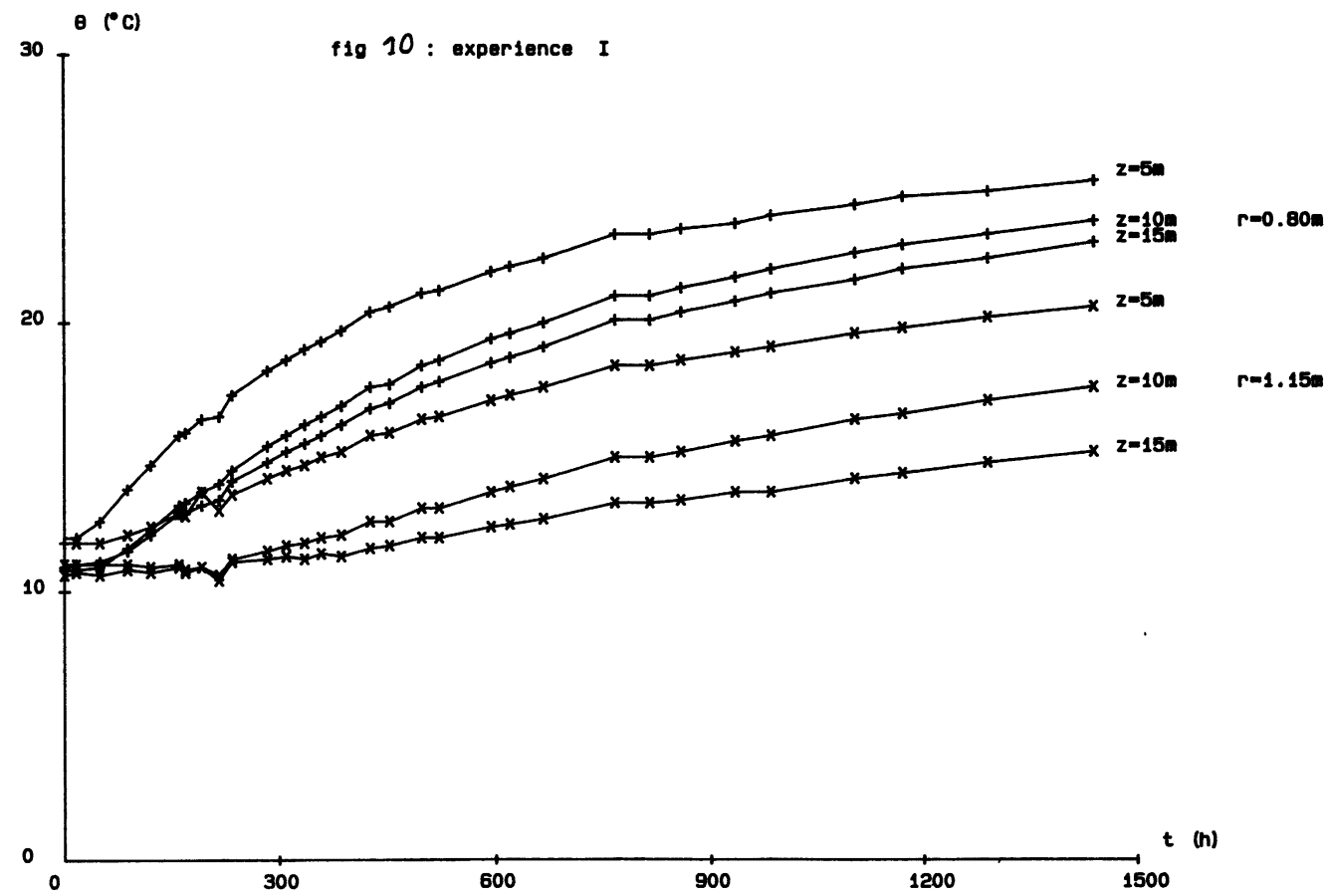

Fig. 10. - Premier stockage.

[First storage.]

$57{ }^{\circ} \mathrm{C}$ après 550 heures de fonctionnement. Nous observons initialement une inversion du gradient thermique vertical par rapport à la première manipulation, lequel redevient très rapidement orienté vers le haut. Puis, nous constatons un tassement des températures du sol à $0,80 \mathrm{~m}$, c'est-à-dire un affaiblissement du gradient thermique vertical, lequel est beaucoup moins sensible à $1,20 \mathrm{~m}$ (Fig. 11).
5.3 Calage du modèle AVEC l'EXPÉRIENCE. Le calage des paramètres a été effectué d'une part sur la première phase de chauffage-relaxation, et d'autre part sur la deuxième phase de chauffage seul. Dans les deux cas, nous présentons l'évolution des températures, expérimentale et théorique, du sol aux rayons $0,80 \mathrm{~m}$ et $1,20 \mathrm{~m}$ et à la profondeur $10 \mathrm{~m}$ en utilisant la méthode de Gaver et le principe 


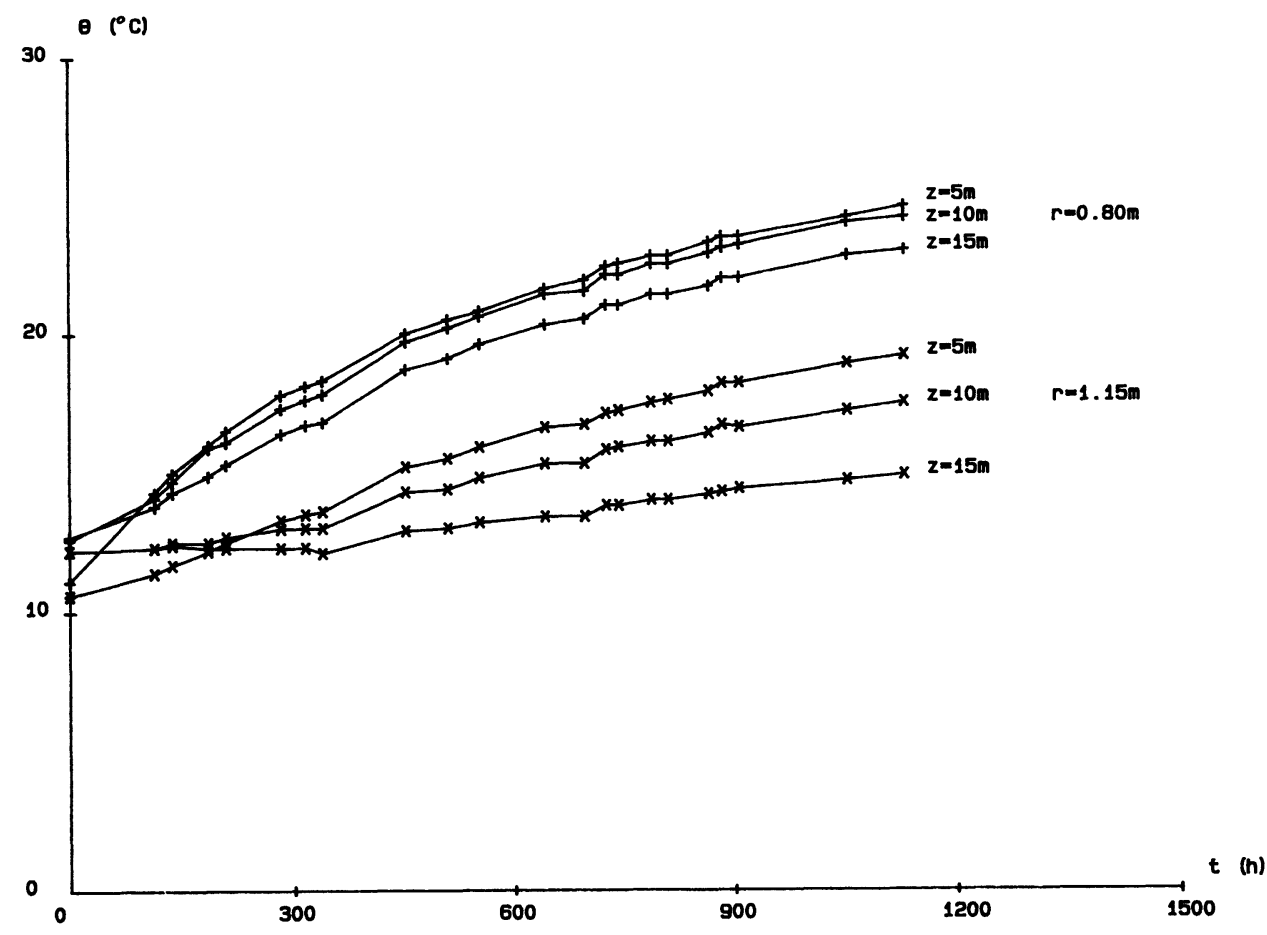

Fig. 11. - Second stockage.

[Second storage.]

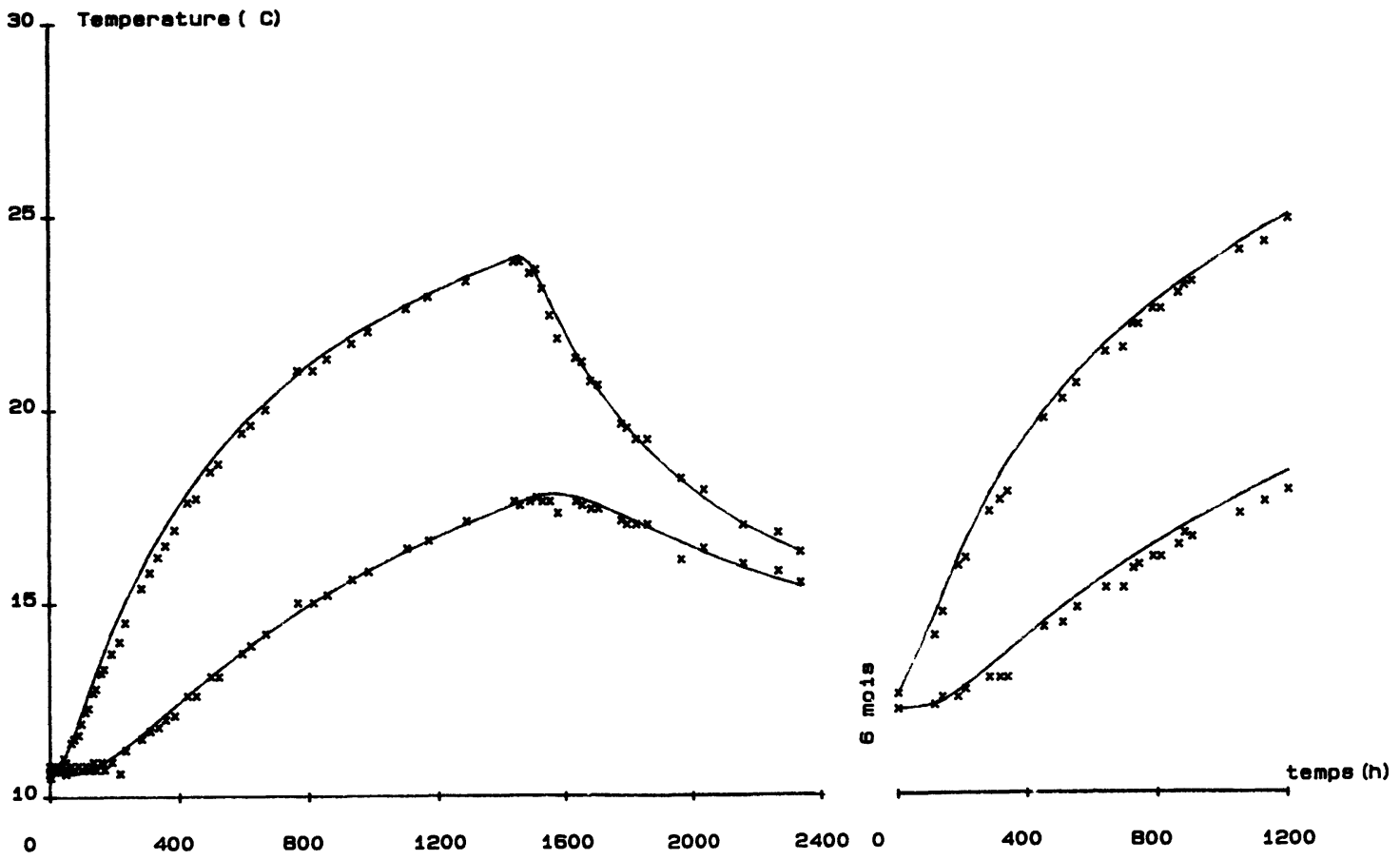

Fig. 12. - Calage du modèle avec l'expérience.

[Adjustement of the model with experimentation.] 
de superposition, ce qui permet de s'affranchir de l'imperfection du créneau de température dans l'annulaire. Cette cote a été choisie comme référence à cause des hétérogénéités constatées en profondeur en tenant compte du fait que la surface est suffisamment éloignée pour que le climat extérieur n'influe pas sur le phénomène [8].

Pour tenir compte des hétérogénéités du sol, nous prenons deux valeurs différentes de la diffusivité à $0,80 \mathrm{~m}$ et $1,20 \mathrm{~m}$, lesquelles correspondent à des diffusivités moyennes équivalentes entre le puits et le point de mesure. Ceci nous donne des diffusivités $a_{1}=0,38 \times 10^{-6} \mathrm{~m}^{2} / \mathrm{s}$ à $0,80 \mathrm{~m}$ et $a_{2}=0,28 \times$ $10^{-6} \mathrm{~m}^{2} / \mathrm{s}$ à $1,20 \mathrm{~m}$ (au lieu de $0,585 \times 10^{-6} \mathrm{~m}^{2} / \mathrm{s}$ pour l'échantillon sec), pour une conductivité sensiblement égale à $\lambda=1,5 \mathrm{~W} / \mathrm{m}$.K. La valeur de cette dernière revêt moins d'importance du fait que nous avons pu observer, par ailleurs, que le champ de température est moins sensible aux variations de la conductivité qu'à celles de la diffusivité.

Sur le tracé, nous constatons une bonne adéquation générale des températures calculées avec celles mesurées pendant la seconde phase, l'erreur n'excédant pas $1^{\circ} \mathrm{C}$ dans le pire des cas (Fig. 12). De plus, nous avons pu constater une diminution très sensible de la diffusivité lorsque la profondeur croît (de l'ordre de $0,03 \times 10^{-6} \mathrm{~m}^{2} / \mathrm{s}$ par mètre, soit $5 \%$ ).

\section{Conclusion.}

De l'étude théorique nous retenons deux modèles permettant de prévoir le champ de température dans le sol et le flux échangé à la paroi :
Le modèle de Gaver est utilisable quelque soit l'instant et le rayon du puits, la seule condition est l'hypothèse de puits infiniment long, mais nous avons vu qu'à $10 \mathrm{~m}$ sous la surface du sol le résultat est déjà très satisfaisant dans le cadre d'un prédimensionnement.

Le modèle dit asymptotique est un peu plus contraignant car il impose en plus des temps suffisamment longs et un rayon de puits assez faible ce qui a l'inconvénient majeur de rendre inadéquat le principe de superposition pour un calcul avec une température d'annulaire variable. Mais par contre, quand ce modèle est utilisable il est beaucoup plus rapide que le précédent.

Quant à l'expérience, nous en tirons quelques remarques :

Nous avons pu constater, sur le site expérimental, des hétérogénéités au sein du milieu stockeur. Il est donc difficile d'évaluer avec précision les paramètres thermophysiques à prendre en compte.

Malgré ces hétérogénéités, le gradient vertical de température reste faible et n'a pas d'incidence sensible sur l'évaluation du stock.

Dans la perspective d'une étude de prédimensionnement, la méthode bidimensionnelle que nous avons mise en œuvre paraît donc suffisante.

Enfin, si l'intérêt du stockage passif par puits unique semble mince en raison de la dégradation rapide du stock dans le temps, la modélisation utilisée s'applique parfaitement au cas d'un stockage actif, c'est-à-dire couplé avec une pompe à chaleur, dont les performances paraissent beaucoup plus favorables.

\section{Bibliographie}

[1] LEROY, G., Etude d'un stockage intersaisonnier de chaleur dans le sol par forages et échangeurs tubulaires verticaux; Rev. Gén. Therm. (1983) p. 151-159.

[2] Sauty, J. P., Projet Corail (1 ${ }^{\mathrm{rc}}$ partie) ; rapport 83 SGN 447 EAU, BRGM Orléans, 1983.

[3] Carslaw, H. S., JaEger, J. C., Conduction of heat transfer in solids (Oxford University Press) 1959.

[4] Ritchie, R. H., Sakakura; A. Y., Asymptotics expansions of solution of the heat conduction equation in internally bounded cylindrical geometry ; J. Appl. Phys. 27 (1956).

[5] Germant, A., Transient temperature around heating pipes maintained at constant temperature, $J$. Appl. Phys. 17 (1946).
[6] Veillon, F., Quelques nouvelles méthodes pour le calcul numérique de la transformée inverse de Laplace; thèse, Université de Grenoble, 11/03/1972.

[7] Nougier, J. P., Méthodes de calcul numérique (éd. Masson, Paris) 1983.

[8] Benet, J. C., Saix, C., Jouanna, P., Etude expérimentale des transferts de masse et d'énergie audessus d'un stockage de chaleur en sol non saturé, Enerstock 85, Toronto.

[9] MAC ADAMs, traduit par Beaufils, Transmission de la chaleur (éd. Dunod, Paris) 1961. 